\title{
Polarimetric studies of carbon stars at high Galactic latitude
}

\author{
A. Goswami and D. Karinkuzhi
}

\author{
Indian Institute of Astrophysics, Koramangala II Block, 560034 Bangalore, India \\ e-mail: aruna@iiap.res.in
}

Received 28 June 2012 / Accepted 21 October 2012

\begin{abstract}
Context. Very little is known about the polarimetric properties of CH stars and carbon-enhanced metal-poor (CEMP) stars, although many of these objects have been studied in detail both photometrically and spectroscopically.

Aims. We aim to derive polarimetric properties for a large sample of CEMP stars and CH stars to fill this gap.

Methods. Multiband polarimetric observations were conducted in the first run for a sample of twenty-nine objects that include twenty-two CEMP and CH stars and seven polarization standards. Estimates of polarization were obtained using standard procedures of polarization calculation.

Results. Five objects in our sample do not show any significant polarization over the different colours of BVRI. For the rest of the objects the derived percentage polarization estimates are $\leq 1 \%$, and they are found to exhibit random behaviour with respect to the inverse of the effective wavelength of observations. Polarization also does not seem to have any correlation with the effective temperatures of the stars.

Conclusions. Our polarimetric estimates indicate there are circumstellar envelopes around these stars that are spherically symmetric or envelopes with little or no dust. In the plane of differential polarization, defined as the difference between the maximum and the minimum polarizations within the $B V R I$-bands, versus their visual magnitude, the stars appear to be confined to a narrow band. The implication of this trend for understanding the nature of the circumstellar environment remains to be determined and requires detailed modelling.
\end{abstract}

Key words. stars: carbon - stars: late-type - stars: variables: general - polarization

\section{Introduction}

Many late-type variables are known to exhibit intrinsic linear polarization due to the scattering of starlight from circumstellar dust distribution in non-spherically symmetric envelopes. However, not much is known about the polarization properties of $\mathrm{CH}$ stars or the carbon-enhanced metal-poor (CEMP) stars, although both photometric and spectroscopic studies do exist for a large portion of these objects. These stars which are distributed in a wide range of Galactic latitudes, are expected to have circumstellar envelopes that give rise to linear polarization. The first-ever estimates of $V$-band polarimetry for a sample of CEMP stars was reported by Goswami et al. (2010a). Within the small sample of stars they found two distinct groups, one with $p \leq 0.4 \%$ and the other with $p \geq 1 \%$, and separation into two groups could be linked to the evolutionary properties of CEMP stars. Time-dependent photometric and polarimetric studies of late-type Mira variables have shown that the decrease in the degree of polarization corresponds to an increase in brightness and vice versa (Dyck 1968). Polarimetric studies of normal carbon stars (Kruszewski \& Gehrels 1968) have also revealed a number of important features. Among these, the most significant are a) a flat wavelength dependence of polarization in yellow to blue; b) time variability in the wavelength dependence of polarization; c) time variability in both the degree of polarization and the position angle; and d) close correlation of polarization with the light variations. It would be useful to examine these properties in the case of CEMP and CH stars. CH stars are known to be radial velocity variables and members of binary systems (McClure 1984; McClure \& Woodsworth 1990). It is known from the literature that stars that show intrinsic polarization are known variable stars; however, the converse statement may not be true, and not all variable stars may show intrinsic polarization.

Among the CEMP stars, the largest fraction is characterized by abundance patterns of neutron-capture elements (CEMP-s stars) that are compatible with the s-process in asymptotic giant branch (AGB) stars. Such abundance patterns are also characteristics of those $\mathrm{CH}$ stars that are members of the Galactic halo (Hartwick \& Cowley 1985). Abundances of carbon, nitrogen, and s-process elements are greatly enhanced in $\mathrm{CH}$ stars with $[\mathrm{C}+\mathrm{N} / \mathrm{Fe}] \geq 1$ (Vanture 1992). The enhancement of s-process elements are greater for the heavy s-process peak elements $(\mathrm{Ba}$ through $\mathrm{Sm}$ ) than for the light s-process peak elements $\mathrm{Sr}, \mathrm{Y}$, and Zr. Orbital data, the abundances of $\mathrm{C}, \mathrm{N}, \mathrm{O}$, and carbon isotopic ratios, are consistent with a binary picture in which $\mathrm{C}$ and s-process elements are transferred onto the $\mathrm{CH}$ star from the AGB star (Vanture 1992), where the ${ }^{13} \mathrm{C}(\alpha, \mathrm{n}){ }^{16} \mathrm{O}$ reaction acts as the source of neutrons for the s-process. Radial velocity variations imply that CEMP-s stars are also members of binary systems (Preston \& Sneden 2000; Lucatello et al. 2005), and a production mechanism similar to the one applicable to $\mathrm{CH}$ stars is believed to hold good for CEMP-s stars (Masseron et al. 2010, and references therein).

The identification of $\mathrm{CH}$ stars and CEMP-s stars as the same class has important implications for certain areas, such as the AGB nucleosynthesis and Galactic enrichment of heavy elements due to low-mass metal-poor stars. Polarimetric properties could reflect upon the nature of the circumstellar environment and complement spectroscopic observations. In particular, polarization, a characteristic property of stars evolving from the red giant stage to planetary nebula, can be used as an important indicator of stellar evolution. Despite the potential usefulness, 
Table 1. Photometric parameters of programme stars.

\begin{tabular}{|c|c|c|c|c|c|c|c|c|c|}
\hline Star & RA(2000) & $\operatorname{Dec}(2000)$ & $l$ & $b$ & V & $J$ & $H$ & $K$ & Dt. of obs. \\
\hline $\mathrm{BD}+59 \mathrm{~d} 389^{*}$ & 020242.08 & +601526.45 & 131.6653 & -1.4005 & 9.07 & 6.535 & 6.203 & 6.023 & 02.02 .2011 \\
\hline HE $0310+0059$ & 031256.91 & +011109.70 & 178.9527 & -45.730 & 11.56 & 9.871 & 9.296 & 9.196 & 01.02 .2011 \\
\hline HE $0518-2322$ & 052035.57 & -231914.31 & 225.6163 & -29.7391 & 12.78 & 11.151 & 10.672 & 10.568 & 02.02 .2011 \\
\hline HE 0519-2053 & 052154.41 & -205035.40 & 223.0620 & -28.6177 & 13.70 & 11.965 & 11.399 & 11.296 & $\begin{array}{l}01.02 .2011 \\
02.02 .2011\end{array}$ \\
\hline HD $43384^{*}$ & 061658.70 & +234427.27 & 187.9943 & 3.5289 & 6.29 & 5.187 & 5.093 & 4.975 & $\begin{array}{l}01.02 .2011 \\
29.03 .2011\end{array}$ \\
\hline HD 55496 & 071211.37 & -225900.61 & 235.7353 & -5.9670 & 8.40 & 6.590 & 6.043 & 5.931 & 28.03.2011 \\
\hline HD $65583^{*}$ & 080032.12 & +291244.48 & 192.1574 & 26.950 & 6.94 & 5.539 & 5.170 & 5.095 & 28.03.2011 \\
\hline HE 0915-0327 & 091808.23 & -033956.69 & 235.2581 & 30.0879 & 12.90 & 9.969 & 8.989 & 8.609 & 28.03.2011 \\
\hline HE 0916-0037 & 091847.65 & -005034.56 & 232.6277 & 31.7927 & 12.80 & 11.226 & 10.782 & 10.658 & 01.02 .2011 \\
\hline HD 81192 & 092445.33 & +194711.86 & 210.2033 & 42.3555 & 6.53 & 4.846 & 4.282 & 4.119 & 2011 \\
\hline HD 90508* & 102803.88 & +484705.64 & 165.1026 & 54.9211 & 6.44 & 5.20 & 4.890 & 4.870 & 2011 \\
\hline HE $1030-1518$ & 103310.06 & -153350.26 & 260.6244 & 35.7077 & 12.06 & 10.375 & 9.801 & 9.657 & 2011 \\
\hline HD 92545 & 104057.70 & -121144.21 & 259.8507 & 39.5218 & 8.56 & 7.548 & 7.347 & 7.282 & 2011 \\
\hline HD $94851^{*}$ & 105644.25 & -203952.62 & 269.8199 & 34.7282 & 9.27 & 8.869 & 8.816 & 8.771 & $\begin{array}{l}02.02 .2011 \\
29.03 .2011\end{array}$ \\
\hline HE $1056-1855$ & 105912.25 & -191106.99 & 269.4844 & 36.2935 & 12.49 & 10.784 & 10.249 & 10.090 & 02.02.2011 \\
\hline HD 100764 & 113542.74 & -143536.66 & 276.8590 & 44.4108 & 8.73 & 7.048 & 6.600 & 6.153 & 02.02 .2011 \\
\hline LEE 107 & 115539.74 & +123448.0 & 258.2830 & 70.4485 & 11.3 & 7.709 & 6.853 & 6.560 & 02.02.2011 \\
\hline HD 107574 & 122151.86 & -182400.15 & 293.1743 & 43.9107 & 8.55 & 7.660 & 7.460 & 7.415 & 28.03.2011 \\
\hline GD 319* & 125005.00 & +550600.00 & 123.3446 & 62.0271 & 12.32 & 11.548 & 11.087 & 11.056 & 02.02 .2011 \\
\hline HD 111721 & 125125.19 & -132928.17 & 302.9257 & 49.3806 & 7.97 & 6.347 & 5.898 & 5.786 & 28.03.2011 \\
\hline HD 112869 & 125922.64 & +374903.69 & 114.5490 & 79.1809 & 9.06 & 6.265 & 5.543 & 5.241 & 29.03.2011 \\
\hline HE 1345-2616 & 134802.08 & -263112.40 & 318.3520 & 34.6512 & - & 9.855 & 9.276 & 9.099 & 29.03.2011 \\
\hline HD 121447 & 135546.96 & -181456.49 & 323.6762 & 42.0161 & 7.81 & 5.397 & 4.442 & 4.282 & 29.03 .2011 \\
\hline HD 125079 & 141720.71 & -041557.81 & 339.6595 & 52.3705 & 8.67 & 7.136 & 6.759 & 6.610 & 29.03 .2011 \\
\hline HD 126681 & 142724.91 & -182440.44 & 332.6355 & 38.8618 & 9.32 & 8.044 & 7.709 & 7.631 & 29.03 .2011 \\
\hline HE $1428-1950$ & 143059.39 & -200341.90 & 332.6046 & 37.0084 & 11.86 & 9.988 & 9.469 & 9.318 & 01.02 .2011 \\
\hline HE 1429-1411 & 143240.57 & -142505.61 & 336.6322 & 41.7343 & 11.10 & 7.622 & 6.721 & 6.346 & 28.03.2011 \\
\hline HD 160529* & 174159.02 & -333013.71 & 355.7023 & -1.7325 & 6.77 & 3.547 & 3.056 & 2.790 & $\begin{array}{l}28.03 .2011 \\
29.03 .2011\end{array}$ \\
\hline HD 164922 & 180230.8624 & +261846.80 & 52.2980 & 21.7853 & 6.99 & 5.550 & 5.20 & 5.110 & 29.03.2011 \\
\hline
\end{tabular}

Notes. ${ }^{(*)}$ Indicates polarization standard star.

almost no polarimetric data currently exists on $\mathrm{CH}$ and CEMP stars. It would be useful to know how these objects compare in terms of their circumstellar environment using polarization properties. It is towards this goal that we have undertaken to study a selected sample of these objects through polarimetric observations.

In the case of stars that are components of binary systems, as is believed to be the case for CH stars and CEMP-s stars, it is reasonable to expect consequent asymmetries or inhomogeneities in the circumstellar envelope, hence a measurable net polarization. We have carried out the BVRI polarimetric studies for a sample of stars comprising a few $\mathrm{CH}$ stars selected from the $\mathrm{CH}$ star catalogue of Bartkevicius (1996) and a few CEMP stars from Christlieb et al. (2001). Unlike the normal carbon stars, with respect to the inverse of the effective wavelength of observation, the polarimetric estimates of these objects are found to show random behaviour. However, the differential polarization, defined as the difference between the observed maximum and minimum polarization within the $B V R I$-bands with respect to their visual magnitudes, is found to exhibit similar bahaviour by the CEMP stars and the $\mathrm{CH}$ stars. The implication of this observed trend in understanding the nature of the circumstellar environment remains to be determined and requires detailed modelling.

In Sect. 2, we present observations and data reduction procedures. In Sect. 3, we discuss the polarimetric calibration, and in Sect. 4 we present our results and discussions. Conclusions are drawn in Sect. 5.

\section{Observations and data reduction}

The sample of stars observed are listed in Table 1 along with several physical and photometric parameters for each star. BVRI polarimetric observations were carried out using the polarimeter attached to the 2-m telescope at IGO, Girawali of IUCAA, Pune during February to March 2011. In addition to the programme stars, a few standard polarization and zero-polarization stars were observed for polarimetric calibrations. The polarimeter has a rotating half wave plate (HWP) and Wollaston prism through which light passes before forming a pair of images of an object on the CCD (Sen \& Tandon 1994; Ramaprakash et al. 1998). The HWP can rotate in several discrete steps, such that its fast axis makes angles $(\alpha)$ with some reference direction (generally celestial north-south). The light transmitting out of the Wollaston prism forms two images of the source on the CCD, with the ordinary and extraordinary set of rays. The observations for each object were taken at four different positions of the HWP: $0^{0}, 22.5^{\circ}, 45^{\circ}$, and $67.5^{\circ}$. Data reduction was carried out using various tasks in IRAF. The task PHOT in APPHOT was used to measure the stellar flux.

\section{Polarimetric calibration}

Three polarized standard and four unpolarized standard stars were observed during the observing run for polarimetric calibration. The estimated $p \%$ for the polarization standards are listed 
Table 2. Polarimetry of polarization standard stars.

\begin{tabular}{|c|c|c|c|c|c|c|c|}
\hline Star names & $\begin{array}{l}\text { HJD } \\
\text { (2) }\end{array}$ & $\begin{array}{c}\text { Filter } \\
\text { (3) }\end{array}$ & $\begin{array}{c}p \\
(\%) \\
(4)\end{array}$ & $\begin{array}{c}\theta \\
\text { (degree) } \\
(5)\end{array}$ & $\begin{array}{c}p \\
(\%) \\
(6)\end{array}$ & $\begin{array}{c}\theta \\
\text { (degree) } \\
(7)\end{array}$ & $\begin{array}{c}\text { Reference } \\
\text { (8) }\end{array}$ \\
\hline \multirow[t]{4}{*}{ HD 43384} & 2455594.31754 & $B$ & $2.92 \pm 0.05$ & 167.4 & & & \\
\hline & & V & $2.80 \pm 0.06$ & 172.6 & 3.0 & 170.0 & Serkowski (1975) \\
\hline & & $R$ & $2.91 \pm 0.08$ & 169.8 & & & \\
\hline & & $I$ & $2.34 \pm 0.07$ & 169.3 & & & \\
\hline \multirow[t]{4}{*}{ HD 43384} & 2455650.12855 & $B$ & $3.10 \pm 0.07$ & 164.3 & & & \\
\hline & & $V$ & $2.78 \pm 0.08$ & 172.2 & & & \\
\hline & & $R$ & $2.81 \pm 0.08$ & 170.9 & & & \\
\hline & & $I$ & $2.53 \pm 0.11$ & 171.9 & & & \\
\hline \multirow[t]{4}{*}{ HD 90508} & 2455594.33936 & $B$ & $0.14 \pm 0.06$ & & & & \\
\hline & & $V$ & $0.03 \pm 0.08$ & & & & \\
\hline & & $R$ & $0.08 \pm 0.08$ & & & & \\
\hline & & $I$ & $0.11 \pm 0.07$ & & & & \\
\hline GD 319 & 2455594.51665 & $V$ & $0.06 \pm 0.04$ & & $0.04 \pm 0.04$ & & Turnshek et al. (1990) \\
\hline \multirow[t]{4}{*}{$\mathrm{BD}+59 \mathrm{~d} 389$} & 2455595.08535 & $\bar{B}$ & $6.79 \pm 0.12$ & 92.6 & & & \\
\hline & & $V$ & $6.52 \pm 0.05$ & 101.7 & $6.67 \pm 0.03$ & 98.2 & Turnshek et al. (1990) \\
\hline & & $R$ & $6.40 \pm 0.05$ & 99.93 & & & \\
\hline & & $I$ & $5.69 \pm 0.05$ & 99.02 & & & \\
\hline \multirow[t]{4}{*}{ HD 94851} & 2455595.28591 & $B$ & $0.09 \pm 0.08$ & & & & \\
\hline & & V & $0.09 \pm 0.10$ & & $0.06 \pm 0.02$ & & Turnshek et al. (1990) \\
\hline & & $R$ & $0.21 \pm 0.12$ & & & & \\
\hline & & $I$ & $0.19 \pm 0.18$ & & & & \\
\hline \multirow[t]{4}{*}{ HD 94851} & 2455650.19658 & $B$ & $0.10 \pm 0.05$ & & & & \\
\hline & & V & $0.09 \pm 0.06$ & & & & \\
\hline & & $R$ & $0.06 \pm 0.06$ & & & & \\
\hline & & $I$ & $0.24 \pm 0.07$ & & & & \\
\hline HD 94851 & 2454950.23292 & V & & & $0.16 \pm 0.28$ & & Goswami et al. (2010a) \\
\hline HD 94851 & 2454952.13280 & $V$ & & & $0.11 \pm 0.12$ & & Goswami et al. (2010a) \\
\hline \multirow[t]{4}{*}{ HD 65583} & 2455649.09875 & $B$ & $0.06 \pm 0.08$ & & & & \\
\hline & & $V$ & $0.05 \pm 0.06$ & & & & \\
\hline & & $R$ & $0.07 \pm 0.24$ & & & & \\
\hline & & $I$ & $0.09 \pm 0.09$ & & & & \\
\hline \multirow[t]{4}{*}{ HD 160529} & 2455649.49125 & $B$ & $7.46 \pm 0.04$ & 20.1 & 7.24 & & Clarke (1998) \\
\hline & & $V$ & $7.76 \pm 0.07$ & 20.4 & 7.52 & 20.1 & Clarke (1998) \\
\hline & & $R$ & $7.41 \pm 0.06$ & 21.7 & & & \\
\hline & & $I$ & $5.49 \pm 0.03$ & 21.1 & & & \\
\hline \multirow[t]{4}{*}{ HD 160529} & 2455650.47868 & $B$ & $7.36 \pm 0.10$ & 19.8 & 6.97 & & Hsu \& Breger (1982) \\
\hline & & V & $7.50 \pm 0.12$ & 18.7 & 7.31 & & Hsu \& Breger (1982) \\
\hline & & $R$ & $7.41 \pm 0.05$ & 21.6 & & & \\
\hline & & $I$ & $4.88 \pm 0.03$ & 21.4 & & & \\
\hline HD 160529 & 2454952.46513 & $V$ & & & $7.46 \pm 0.02$ & 20.9 & Goswami et al. (2010a) \\
\hline HD 160529 & & & & & $7.35 \pm 0.55$ & & Reiz \& Franco (1998) \\
\hline HD 160529 & & & & & $7.3 \pm 0.54$ & 20 & Serkowski (1975) \\
\hline
\end{tabular}

Notes. Our estimates are listed in Cols. (4) and (5). Estimates listed in Cols. (6) and (7) are from references listed in Col. (8).

in Table 2 along with their literature values whenever available. We find close agreement between our estimates and the literature values. For the standard polarized stars, the position angles $(\theta)$ are listed in Col. 5 of Table 2. For stars with null polarization the position angles are not defined, hence position angles are only presented for stars that give non-zero polarization.

The errors in the polarization measurements are primarily by dominated photon noise. For low values of polarization, the number of photo-electrons corresponding to ordinary and extraordinary images are approximately equal. The error in the polarization measurements are given by $\left(\frac{\sqrt{N+s N_{\mathrm{b}}}}{2 N} \times 100 \%\right)$ where $s=\pi r^{2}$, " $r$ " is the radius of the aperture used to find the total flux of the star image (in pixels), $N$ is the total source counts, and $N_{\mathrm{b}}$ the average background sky count per pixel. The estimates of the percentage polarization ( $p \%)$, along with the corresponding error estimates, are listed in Table 3. For the majority of the objects the measured errors are $\leq 0.1 \%$.
The zero-polarization, standard stars were observed to check for any possible instrumental error, which proved to be $\sim 0.1 \%$. As noted from Table 2, the amount of polarization for the standard unpolarized stars HD 90508, HD 94851, HD 65583, and GD 319 are non-zero. It is reasonable to expect the amount of polarization determined from a single measurement of a standard star to lie within the limits $0.0 \leq p \% \leq 0.21$ (Dyck \& Jennings 1971). Following this criterion the objects with $p \leq 0.2 \%$ are considered to be unpolarized at the epoch of observation.

\section{Results and discussions}

Close agreement between our polarimetric estimates for the polarized and zero-polarization standard stars with those from the literature lends support to the reliability of our results. Estimated $p \%$ for the programme stars listed in Table 3 have not been corrected for interstellar polarization. The interstellar reddening listed for the programme stars is also low. For objects at low 
Table 3. BVRI polarimetry of carbon stars.

\begin{tabular}{|c|c|c|c|c|c|c|}
\hline Star names & $E(B-V)$ & Reference & HJD & Filter & $\begin{array}{c}p \\
(\%)\end{array}$ & $\begin{array}{c}\theta \\
\text { (degree) }\end{array}$ \\
\hline HE $0310+0059$ & - & & 2455594.16819 & $\begin{array}{l}B \\
V \\
R \\
I\end{array}$ & $\begin{array}{c}0.77 \pm \mathbf{0 . 1 0} \\
\mathbf{0 . 8 9} \pm \mathbf{0 . 0 7} \\
\mathbf{0 . 7 0} \pm \mathbf{0 . 0 7} \\
\mathbf{0 . 5 8} \pm \mathbf{0 . 0 9}\end{array}$ & $\begin{array}{l}130.1 \\
130.9 \\
142.1 \\
147.4\end{array}$ \\
\hline HE 0518-2322 & 0.028 & 1 & 2455595.25775 & $\begin{array}{l}B \\
V \\
R \\
I\end{array}$ & $\begin{array}{l}\mathbf{0 . 3 9} \pm \mathbf{0 . 1 2} \\
\mathbf{0 . 3 3} \pm \mathbf{0 . 0 6} \\
0.33 \pm 0.14 \\
0.73 \pm 0.19\end{array}$ & $\begin{array}{c}147.5 \\
177.9 \\
144.3 \\
26.2\end{array}$ \\
\hline HE 0519-2053 & - & & 2455595.21464 & $\begin{array}{l}B \\
V \\
R \\
I\end{array}$ & $\begin{array}{c}\mathbf{0 . 5 7} \pm \mathbf{0 . 1 3} \\
0.28 \pm 0.13 \\
\mathbf{0 . 2 1} \pm \mathbf{0 . 0 7} \\
0.28 \pm 0.16\end{array}$ & $\begin{array}{l}159.9 \\
172.9 \\
147.0 \\
147.5\end{array}$ \\
\hline HE 0915-0327 & 0.041 & 1 & 2455649.31148 & $\begin{array}{l}B \\
V \\
R \\
I\end{array}$ & $\begin{array}{l}0.75 \pm 0.32 \\
\mathbf{0 . 5 2} \pm \mathbf{0 . 0 7} \\
\mathbf{0 . 4 4} \pm \mathbf{0 . 0 8} \\
\mathbf{0 . 7 7} \pm \mathbf{0 . 0 8}\end{array}$ & $\begin{array}{c}147.5 \\
14.3 \\
147.4 \\
9.4\end{array}$ \\
\hline $\begin{array}{l}\text { HE 0915-0327 } \\
\text { HE 0916-0037 }\end{array}$ & $\begin{array}{l}0.041 \\
0.030\end{array}$ & $\begin{array}{l}1 \\
1\end{array}$ & $\begin{array}{l}2455649.31148 \\
2455594.40375\end{array}$ & $\begin{array}{l}B \\
B \\
V \\
R \\
I\end{array}$ & $\begin{array}{l}0.75 \pm 0.32 \\
0.46 \pm 0.20 \\
0.19 \pm 0.08 \\
0.25 \pm 0.09 \\
0.10 \pm 0.11\end{array}$ & $\begin{array}{c}147.5 \\
12.5 \\
127.5\end{array}$ \\
\hline HE $1030-1518$ & 0.065 & 1 & 2455650.26381 & $\begin{array}{l}B \\
V \\
R \\
I\end{array}$ & $\begin{array}{l}\mathbf{0 . 5 1} \pm \mathbf{0 . 0 6} \\
0.18 \pm 0.09 \\
\mathbf{0 . 4 9} \pm \mathbf{0 . 0 8} \\
\mathbf{0 . 4 6} \pm \mathbf{0 . 0 7}\end{array}$ & $\begin{array}{c}17.0 \\
\\
25.3 \\
129.3\end{array}$ \\
\hline HE $1056-1855$ & 0.045 & 1 & 2455595.35264 & $\begin{array}{l}B \\
V \\
R \\
I\end{array}$ & $\begin{array}{l}0.08 \pm 0.08 \\
0.16 \pm 0.13 \\
0.19 \pm 0.11 \\
0.19 \pm 0.11\end{array}$ & \\
\hline HE $1345-2616$ & - & & 2455650.39694 & $\begin{array}{l}B \\
V \\
R \\
I\end{array}$ & $\begin{array}{l}\mathbf{0 . 4 3} \pm \mathbf{0 . 0 5} \\
\mathbf{0 . 4 7} \pm \mathbf{0 . 0 6} \\
\mathbf{0 . 4 2} \pm \mathbf{0 . 0 7} \\
0.29 \pm 0.10\end{array}$ & $\begin{array}{l}153.9 \\
148.5 \\
163.7 \\
134.2\end{array}$ \\
\hline HE $1428-1950$ & 0.044 & 1 & 2455594.49870 & $\begin{array}{l}B \\
V \\
R \\
I\end{array}$ & $\begin{array}{l}0.34 \pm 0.15 \\
\mathbf{0 . 8 7} \pm \mathbf{0 . 0 7} \\
\mathbf{0 . 8 7} \pm \mathbf{0 . 1 2} \\
\mathbf{0 . 7 0} \pm \mathbf{0 . 1 3}\end{array}$ & $\begin{array}{l}179.0 \\
150.2 \\
152.3 \\
150.8\end{array}$ \\
\hline HD 1429-1411 & - & & 2455649.45900 & $\begin{array}{l}B \\
V \\
R \\
I\end{array}$ & $\begin{array}{l}\mathbf{0 . 6 9} \pm \mathbf{0 . 0 8} \\
\mathbf{0 . 9 0} \pm \mathbf{0 . 0 5} \\
0.67 \pm 0.26 \\
\mathbf{0 . 5 6} \pm \mathbf{0 . 0 4}\end{array}$ & $\begin{array}{c}20.0 \\
139.6 \\
126.9 \\
129.7\end{array}$ \\
\hline HD 55496 & 0.05 & 2 & 2455649.13837 & $\begin{array}{l}B \\
V \\
R \\
I\end{array}$ & $\begin{array}{l}\mathbf{0 . 3 2} \pm \mathbf{0 . 0 9} \\
\mathbf{0 . 1 8} \pm \mathbf{0 . 0 6} \\
0.03 \pm 0.07 \\
0.16 \pm 0.07\end{array}$ & 167.9 \\
\hline HD 81192 & 0.0 & 3 & 2455649.23910 & $\begin{array}{l}B \\
V \\
R \\
I\end{array}$ & $\begin{array}{l}0.14 \pm 0.08 \\
0.17 \pm 0.09 \\
0.09 \pm 0.06 \\
\mathbf{0 . 1 6} \pm \mathbf{0 . 0 5}\end{array}$ & \\
\hline HD 92545 & 0.014 & 4 & 2455649.21908 & $\begin{array}{l}B \\
V \\
R \\
I\end{array}$ & $\begin{array}{l}0.08 \pm 0.09 \\
0.02 \pm 0.08 \\
0.05 \pm 0.08 \\
0.09 \pm 0.13\end{array}$ & \\
\hline HD 100764 & 0.02 & 5 & 2455595.37625 & $\begin{array}{l}B \\
V \\
R \\
I\end{array}$ & $\begin{array}{l}0.12 \pm 0.08 \\
0.16 \pm 0.11 \\
0.28 \pm 0.13 \\
0.37 \pm 0.14\end{array}$ & $\begin{array}{l}137.2 \\
24.1\end{array}$ \\
\hline LEE 107 & - & & 2455595.40695 & $\begin{array}{l}B \\
V \\
R \\
I\end{array}$ & $\begin{array}{l}0.07 \pm 0.09 \\
0.16 \pm 0.11 \\
0.22 \pm 0.13 \\
0.31 \pm 0.15\end{array}$ & $\begin{array}{l}25.7 \\
16.9\end{array}$ \\
\hline
\end{tabular}


Table 3. continued.

\begin{tabular}{|c|c|c|c|c|c|c|}
\hline Star names & $E(B-V)$ & Reference & HJD & Filter & $\begin{array}{c}p \\
(\%)\end{array}$ & $\begin{array}{c}\theta \\
\text { (degree) }\end{array}$ \\
\hline HD 107574 & 0.012 & 4 & 2455649.36574 & $\begin{array}{l}B \\
V \\
R \\
I\end{array}$ & $\begin{array}{l}0.05 \pm 0.06 \\
0.07 \pm 0.07 \\
0.14 \pm 0.11 \\
0.19 \pm 0.16\end{array}$ & \\
\hline HD 111721 & 0.01 & 6 & 2455649.39645 & $\begin{array}{l}B \\
V \\
R \\
I\end{array}$ & $\begin{array}{l}0.28 \pm 0.10 \\
\mathbf{0 . 2 2} \pm \mathbf{0 . 0 4} \\
\mathbf{0 . 2 7} \pm \mathbf{0 . 0 7} \\
0.17 \pm 0.07\end{array}$ & $\begin{array}{l}167.6 \\
147.0 \\
179.0\end{array}$ \\
\hline HD 112869 & 0.0 & 5 & 2455650.29685 & $\begin{array}{l}B \\
V \\
R \\
I\end{array}$ & $\begin{array}{l}0.13 \pm 0.07 \\
\mathbf{0 . 2 9} \pm \mathbf{0 . 0 8} \\
0.15 \pm 0.12 \\
0.05 \pm 0.10\end{array}$ & 147.5 \\
\hline HD 121447 & 0.05 & 7 & 2455650.33307 & $\begin{array}{l}B \\
V \\
R \\
I\end{array}$ & $\begin{array}{l}0.29 \pm 0.12 \\
\mathbf{0 . 6 0} \pm \mathbf{0 . 0 9} \\
\mathbf{0 . 5 3} \pm \mathbf{0 . 0 7} \\
\mathbf{0 . 4 6} \pm \mathbf{0 . 0 9}\end{array}$ & $\begin{array}{l}147.5 \\
166.7 \\
166.2 \\
165.7\end{array}$ \\
\hline HD 125079 & 0.0 & 8 & 2455650.42737 & $\begin{array}{l}B \\
V \\
R \\
I\end{array}$ & $\begin{array}{l}0.28 \pm 0.13 \\
0.14 \pm 0.07 \\
0.37 \pm 0.13 \\
0.17 \pm 0.17\end{array}$ & $\begin{array}{l}152.5 \\
139.8\end{array}$ \\
\hline HD 126681 & 0.0 & 9 & 2455650.45205 & $\begin{array}{l}B \\
V \\
R \\
I\end{array}$ & $\begin{array}{l}0.07 \pm 0.07 \\
0.06 \pm 0.08 \\
0.18 \pm 0.10 \\
0.15 \pm 0.14\end{array}$ & \\
\hline HD 164922 & 0.0 & 3 & 2455649.50958 & $\begin{array}{l}B \\
V \\
R\end{array}$ & $\begin{array}{l}0.28 \pm 0.12 \\
0.28 \pm 0.10 \\
0.15 \pm 0.08\end{array}$ & $\begin{array}{c}17.5 \\
165.3\end{array}$ \\
\hline
\end{tabular}

References. 1) Beers et al. (2007); 2) Beers et al. (2000); 3) Masseron et al. (2010); 4) Allen \& Barbuy (2006a); 5) Eggen (1972); 6). Ryan \& Lambert (1995); 7) Bergeat et al. (1999); 8) Smith et al. (1993); 9) Melendez et al. (2010). Estimates with $p \% \geq 3 \sigma$ are indicated using boldface.

Galactic latitude, intrinsically polarized stars may have a component of interstellar polarization that is negligible for objects at high Galactic latitude. Estimated errors in $p \%$ are photon-noisedominated and negligibly small in the case of very bright objects.

Taking the error estimates into account, the derived percentage polarization is above the $3 \sigma$ limit in all four bands for only one object, HE $0310+0059$. Seven stars exhibit polarization above the $3 \sigma$ limit in three bands, three stars in two bands, and two stars in a single band. Nine objects in our sample show polarization at a level below the $3 \sigma$ limit in all four bands. The polarization estimates above the $3 \sigma$ limit are indicated using boldface in Table 3 .

\subsection{Polarimetric characteristics: wavelength dependence of polarization}

The polarization estimates for the programme stars are low $(\leq 1 \%)$ (Table 3). In Fig. 1, we show the response of the $B V R I$ polarimetric estimates for a few selected stars that show polarization above the $3 \sigma$ limit in more than two bands against the corresponding inverse of the effective wavelength of observation. The wavelength dependence of polarization is found to be random in nature and does not follow any systematic pattern. A discussion of the wavelength dependence of polarization is meaningful only in cases where the estimates of percentage polarization are above the $3 \sigma$ limit. Nine objects in our sample, HE 0916-0037, HE 1056-1855, HD 92545, HD 100764, LEE 107, HD 107574, HD 125079, HD 126681, and HD 164922, show polarization below the
$3 \sigma$ limit in all four bands. The object, HE $0310+0059$, that shows significant polarization in all four bands shows a maximum in the $V$-band. The objects HD 81192 and HD 112869 show polarization above the $3 \sigma$ limit only in one band, the $I$ band and $V$ band, respectively. HD 55496, HD 111721, and HE 0519-2053 show polarization above the $3 \sigma$ limit in two bands, $B V, V R$, and $B R$, respectively. Seven objects in our sample, HE 0518-2322, HE 0915-0327, HE 1030-1518, HE 1345-2616, HE 1428-1950, HE 1429-1411, and HD 121447 show polarization above the $3 \sigma$ limit in three bands. While the objects HE 1030-1518, HE 1345-2616, and HE 1428-1950 show a rather flat wavelength dependence of polarization within the errors in $B, R$, and $I$ bands, the objects HE 0518-2322 and HE 0915-0327 show a maximum in $I$ band, and the objects HE 1429-1411, and HD 121447 show a maximum in $V$ band.

As discussed in Zickgraph \& Schulte-Ladbeck (1989), dust particles of different radii and composition can show polarization maximum in different bands. In many cases maximum polarization for scattering of light caused by small dust particles can be observed at short wavelengths. Small carbon particles are known to exist in the circumstellar envelopes of carbon stars. The optical polarization of the evolved carbon star R Scl showed $\mathrm{a} \sim \lambda^{-4}$ wavelength-dependence, attributed to scattering by small amorphous carbon dust grains (Yudin \& Evans 2002) and polarimetric variability was also detected for this object on time-scales from hours to years.

In the upper panel of Fig. 2, we show the $V$-band percentage polarization estimates $\left(p_{v} \%\right)$ of the same stars as shown in 


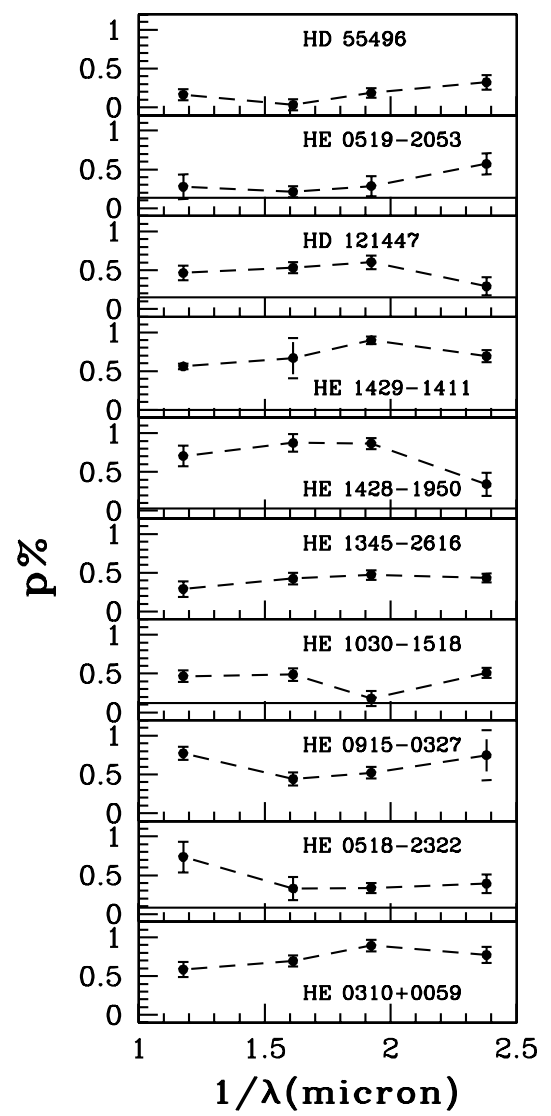

Fig. 1. Response of the observed BVRI percentage polarization estimates with respect to the inverse of the effective wavelength of observation for a sample of CEMP stars and $\mathrm{CH}$ stars. The solid line in each panel indicates the mean interstellar polarization level $(\sim 3 E(B-V))$.

Fig. 1, against the interstellar reddening $E(B-V)$ values whenever available. The mean interstellar polarization $\sim 3 E(B-V)$ (Parthasarathy et al. 2005) is also indicated. The estimated $V$-band percentage polarization is higher than the mean interstellar contribution to the polarization, indicating that they are intrinsically polarized.

Polarization of carbon stars is known to show flatter wavelength dependence than the oxygen-rich stars. A mild wavelength dependence of polarization noticed in some cases is somewhat similar to those reported by Raveendran (1991) for a few carbon stars of Mira variables; i.e., RT Pup shows a systematic increase in polarization towards the red, and X Vel shows higher polarization in the $R$ band than in the $V$ band. Polarimetric studies of some giants and supergiants (Dyck \& Jennings 1971; Lopez \& Hiriart 2011) have shown that the wavelength dependence of polarization varies widely and often changes with time and that the largest changes in the amount of polarization occurs in the ultraviolet. There are, however, exceptional cases of S Per and 119 Tau. In S Per polarization rises abruptly into the ultraviolet but at other times decreases on a timescale of few days (Dyck \& Jennings 1971). In the case of 119 Tau, polarization in the $U$-band was found to be about one-half that of the $V$-band; in addition, the position-angle was found to show changes with wavelength, which they attribute to the presence of more than one component of polarization. Cases where changes in the position angle with wavelength range from almost nothing to as high as 90 degrees are also noted by Dyck \& Jennings (1971). Monitoring time variations of position angles with respect to
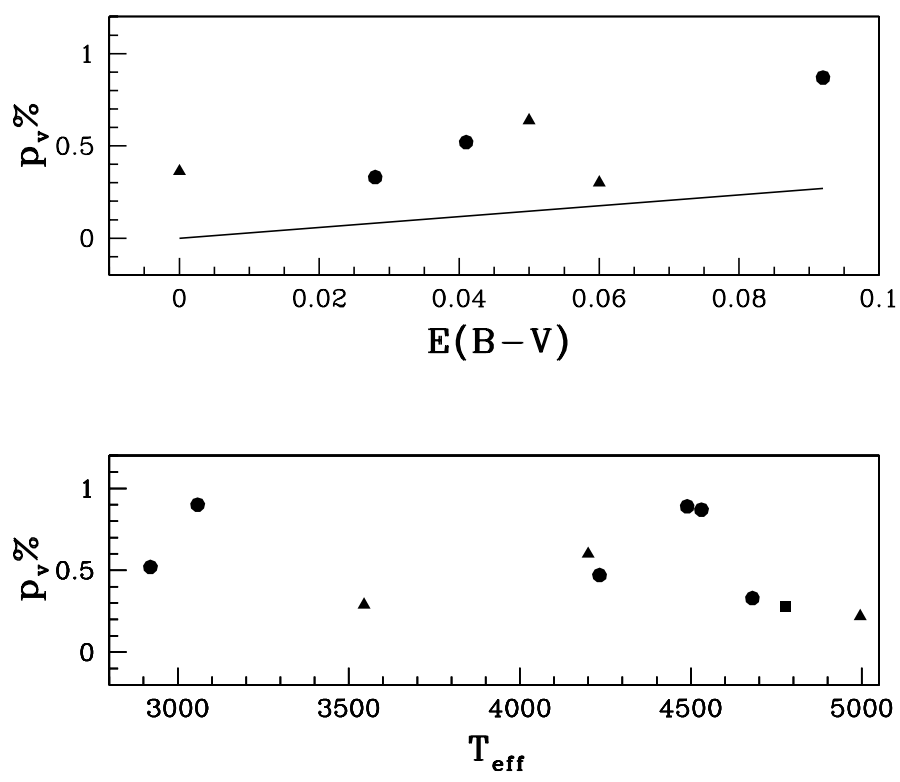

Fig. 2. Top: the observed $p_{v} \%$ vs the interstellar $E(B-V)$. The solid line represents the mean interstellar polarization $(\sim 3 E(B-V))$. $E(B-V)$ values are not available for all ten objects. In all the panels the stars from the $\mathrm{CH}$ star catalogue are represented by solid triangles and the carbon stars from Hamburg/ESO survey by solid circles. The star represented by a solid square corresponds to HE 0519-2053. Bottom: the observed $V$-band percentage polarization $\left(p_{v} \%\right)$ vs the effective temperatures for the same objects as in Fig. 1. Polarization does not seem to have any correlation with temperature.

wavelength would be worthwhile for investigating whether there is a tight relationship between these two parameters.

\subsection{Polarimetric characteristics: temperature dependence of polarization}

The response of the $V$-band polarimetric estimates ( $p_{v} \%$ ) for the programme stars with respect to their effective temperatures is shown in Fig. 2 (lower panel). Observed $p_{v} \%$ estimates do not seem to have any correlation with temperature.

Apart from two objects, HE 0915-0327 and HE 1429-1411 with effective temperatures of $2920 \mathrm{~K}$ and $3058 \mathrm{~K}$, respectively, the temperature for the rest of the CEMP stars ranges from 4200 to $4900 \mathrm{~K}$. The coolest CEMP star in our sample, HE 0915-0327, with $p_{v} \sim 0.5 \%$ shows a maximum polarization in the $I$-band $(0.77 \%)$. Within the sample the highest polarization is seen in HE 1429-1411 with $p_{v} \sim 0.9 \%$; it shows minimum polarization in the $I$-band. The temperature and metallicity ranges for the HD stars are about $3200-6400 \mathrm{~K}$, and 0.05 to -1.6. The hottest objects HD $92545(6240 \mathrm{~K})$ and HD 107574 $(6340 \mathrm{~K})$ do not show any significant polarization in BVRI. The coolest object, HD 112869, shows polarization only in the $V$-band with $p_{v} \sim 0.29 \%$. HD 121447 with $T_{\text {eff }}$ of about $4200 \mathrm{~K}$ returns the highest polarization in the $V$-band among all the HD stars in our sample.

The metallicity data for this sample of stars is scanty; the metallicity estimates are available only for three CEMP stars and nine HD stars in our sample (Table 4), and a fraction of them do not show any significant polarization. To examine the temperature and metallicity dependence of polarization, it would be necessary to consider a larger sample covering a much wider range in temperature and metallicity. Among the objects that 
Table 4. Atmospheric parameters of the programme stars.

\begin{tabular}{|c|c|c|c|c|}
\hline Star names & $\begin{array}{l}T_{\text {eff }} \\
(\mathrm{K})\end{array}$ & $\begin{array}{c}\log g \\
\left(\mathrm{~cm} \mathrm{~s}^{-2}\right)\end{array}$ & $\begin{array}{c}{[\mathrm{Fe} / \mathrm{H}]} \\
(\mathrm{dex})\end{array}$ & Reference \\
\hline HE $0310+0059$ & $4489^{a} / 4861$ & 1.69 & -1.32 & 1 \\
\hline HE 0518-2322 & $4680^{a}$ & - & - & - \\
\hline HE 0519-2053 & 4775 & 1.46 & -1.45 & 1 \\
\hline HE 0915-0327 & $2920^{a}$ & - & - & - \\
\hline HE 0916-0037 & $4872^{a}$ & - & - & - \\
\hline HE $1030-1518$ & $4350^{a}$ & - & - & - \\
\hline HE 1056-1855 & $4280^{a}$ & - & - & - \\
\hline HE 1345-2616 & $4233^{a}$ & - & - & - \\
\hline HE 1428-1950 & 4531 & 0.85 & -2.07 & 1 \\
\hline HE 1429-1411 & $3058^{a}$ & - & - & - \\
\hline HD 55496 & 4800 & 2.8 & -1.55 & 2 \\
\hline HD 81192 & $\begin{array}{l}4705 \\
4582 \\
4755 \\
4755\end{array}$ & $\begin{array}{c}2.50 \\
2.75 \\
2.4 \\
2.4\end{array}$ & $\begin{array}{l}-0.62 \\
-0.70 \\
-0.60 \\
-0.61\end{array}$ & $\begin{array}{l}2 \\
3 \\
4 \\
5\end{array}$ \\
\hline HD 92545 & 6240 & 4.23 & -0.26 & 6 \\
\hline HD 100764 & 4846 & 2.2 & -0.59 & 7 \\
\hline LEE 107 & $3288^{a}$ & - & - & - \\
\hline HD 107574 & $\begin{array}{l}6340 \\
6340 \\
6340\end{array}$ & $\begin{array}{l}3.87 \\
3.87 \\
3.87\end{array}$ & $\begin{array}{l}-0.36 \\
-0.54 \\
-0.16\end{array}$ & $\begin{array}{l}6 \\
6 \\
6\end{array}$ \\
\hline HD 111721 & $\begin{array}{l}4995 \\
4825 \\
4800 \\
5164 \\
4940 \\
5103 \\
5000 \\
4860\end{array}$ & $\begin{array}{c}2.52 \\
2.2 \\
3.00 \\
3.27 \\
2.40 \\
2.87 \\
\\
2.2\end{array}$ & $\begin{array}{l}-1.26 \\
-1.54 \\
-1.68 \\
-0.98 \\
-1.34 \\
-1.25 \\
-1.34 \\
-1.57\end{array}$ & $\begin{array}{c}8 \\
9 \\
10 \\
11 \\
12 \\
13 \\
14 \\
15\end{array}$ \\
\hline HD 112869 & $3545^{a}$ & - & - & - \\
\hline HD 121447 & 4200 & 0.8 & 0.05 & 16 \\
\hline HD 125079 & $\begin{array}{l}5300 \\
5305\end{array}$ & $\begin{array}{l}3.50 \\
3.50\end{array}$ & $\begin{array}{l}-0.16 \\
-0.30\end{array}$ & $\begin{array}{l}17 \\
18\end{array}$ \\
\hline HD 126681 & $\begin{array}{l}5450 \\
5533 \\
5595 \\
5625 \\
5500\end{array}$ & $\begin{array}{c}4.5 \\
4.2 \\
4.43 \\
4.95 \\
4.63\end{array}$ & $\begin{array}{l}-1.25 \\
-1.14 \\
-1.12 \\
-1.09 \\
-1.45\end{array}$ & $\begin{array}{c}9 \\
19 \\
20 \\
11 \\
21\end{array}$ \\
\hline HD 164922 & $5412^{a}$ & - & - & - \\
\hline
\end{tabular}

References. 1) Kennedy et al. (2011); 2) Cennaro et al. (2007); 3) Cottrell \& Sneden (1986); 4) Luck \& Bond (1985); 5) Luck \& Bond (1983); 6) North et al. (1994); 7) Dominy (1984); 8) Gratton \& Sneden (1994); 9) Fulbright (2000); 10) Cavallo et al. (1997); 11) Gratton et al. (1996); 12) Ryan \& Lambert (1995); 13) Gratton \& Sneden (1994); 14) Pilachowski (1993); 15) Francois (1988); 16) Smith (1984); 17) Smith et al. (1993); 18) Smith \& Lambert (1986); 19) Nissen et al. (2000); 20) Nissen \& Schuster (1997); 21) Tomkin et al. (1992); ${ }^{(a)}$ estimated from $(J-K)$ temperature calibrations of Alonso et al. (1996).

show finite polarization, both $\mathrm{CH}$ and CEMP stars show similar responses with respect to $T_{\text {eff }}$.

Simultaneous polarimetric observations in $B V R I$ are limited in the literature. Except for HD 100764 for none of the programme stars' literature values are available. We have presented first-time polarimetric estimates for this set of objects, which would be useful later for time variability studies of polarization and its wavelength dependence, as well as for the time variability of polarization and position angles.

\subsection{Comments on individual stars}

\section{F Str $\lambda 4077$ stars HD 92545 and HD 107574}

These two objects are known to show abnormally strong Sr line at $4077 \AA$ in their spectra. Bidelmann (1981) classified these objects as F Str $\lambda 4077$ stars. They exhibit enhancement of light and heavy s-process elements but abundances of iron-peak elements are similar to those generally seen in F type stars. The radial velocities of HD 92545 and HD 107574 are $-18 \mathrm{~km} \mathrm{~s}^{-1}$ and $-28 \mathrm{~km} \mathrm{~s}^{-1}$, respectively. HD 107574 is said to be a binary (North \& Duquennoy 1991). The estimated distance for HD 92545 is about $101 \mathrm{pc}$ and for HD 107574 the distance ranges between 120-150 pc (North 1987). Based on kinematics and chemical compositions, North \& Duquennoy (1991) suggest that these two objects are probably the main-sequence progenitors of barium stars rather than being population II objects. Estimated $B V R I$ percentage polarization for these two objects are below the $3 \sigma$ limit in all four bands. The near-zero polarization observed in these two objects may be an indication of their early stage of evolution, yet to form aspherical dust shells around them. At the instance of having circumstellar envelopes this would imply they are spherically symmetric.

The subgiant CH stars HD 55496 and HD 125079

HD 55496 and HD 125079 are entered in both the barium star catalogue of Lü (1991) and the CH star catalogue of Bartkevicius (1996) indicating that some uncertainties exist regarding their classification. These two objects are referred to as subgiant CH stars by Smith \& Lambert (1986). A high radial velocity of $\sim+324 \mathrm{~km} \mathrm{~s}^{-1}$ reported by Bond (1974) for HD 55496 is consistent with those generally observed for $\mathrm{CH}$ giants. In view of the close similarities between the spectral peculiarities of the two groups, it was proposed that the subgiant and giant $\mathrm{CH}$ stars form a continuous sequence of stars of the same abnormal chemical composition, differing only in temperature and luminosities (Bond 1974).

\section{HD 100764}

This object is known to have a detached cold dust shell that is optically thin with a relatively large dust grain size (Parthasarathy 1991). HD 100764 is the only example in our sample for which previous polarimetric estimates exist. Multiband photopolarimetry does not show any significant variations in polarization in $B, V, R$, and $I$ bands. A comparison of the polarimetric estimates for this object is presented in Table 5.

HD 121447, HD 111721

The object HD 121447 is reported to exhibit an enhancement of nitrogen abundance (roughly twice solar), slight depletion of oxygen, and near solar or marginal enhancement of carbon abundances (Smith 1984). Abundance peculiarities, such as excess ${ }^{12} \mathrm{C}$ and s-process overabundances observed in this star, are accounted for by assuming that roughly $10^{-2}$ of the outer envelope is contaminated by pure ${ }^{12} \mathrm{C}$ and s-process heavy elements (Smith 1984). $E(B-V)$ for this object is small $\sim 0.05$; the estimated polarizations seem to represent the star's intrinsic polarization property.

The object HD 111721 has been referred to as a metal-poor subgiant star by Cavallo et al. (1997). However, from kinematics, Ryan \& Lambert (1995) confirm it to be a Galactic halo object. Gratton \& Sneden (1991) determine a radial velocity 
Table 5. HD 100764: comparison of polarimetric estimates with literature values.

\begin{tabular}{lcccccccccc}
\hline \hline Star name & $E(B-V)$ & HJD & Filter & $\begin{array}{c}p \\
(\%)\end{array}$ & $\begin{array}{c}\theta \\
(\text { degree })\end{array}$ & HJD & Filter & $\begin{array}{c}p \\
(\%)\end{array}$ & $\begin{array}{c}\theta \\
(\text { degree })\end{array}$ \\
$(1)$ & $(2)$ & $(3)$ & $(4)$ & $(5)$ & $(6)$ & $(7)$ & $(8)$ & $(9)$ & $(10)$ & Reference \\
\hline HD 100764 & 0.02 & 2447971 & $B$ & $0.13 \pm 0.12$ & $127.2 \pm 25.1$ & 2455595.37625 & $B$ & $0.115 \pm 0.0860$ & 143.4 & 1 \\
& & 2447971 & $V$ & $0.20 \pm 0.05$ & & 2455595.37625 & $V$ & $0.163 \pm 0.107$ & 143.4 & 1 \\
& & 2447971 & $R$ & $0.01 \pm 0.04$ & $102.6 \pm 6.0$ & 2455595.37625 & $R$ & $0.278 \pm 0.132$ & 137.2 & 1 \\
& & 2447971 & $I$ & $0.40 \pm 0.08$ & $78.0 \pm 5.5$ & 2455595.37625 & $I$ & $0.372 \pm 0.144$ & 24.1 & 1 \\
& 2448398 & $V$ & $0.42 \pm 0.14$ & & 2454952.14664 & $V$ & $0.330 \pm 0.06$ & 4.0 & 2 \\
\hline
\end{tabular}

Notes. Estimates listed in Cols. (5) and (6) are from Parthasarathy et al. (2005). Our estimates corresponding to the HJDs in Col. (7) are presented in Cols. (9) and (10). In the last column, reference 1 indicates this work and 2 indicates Goswami et al. (2010a).

of $+22.3 \mathrm{~km} \mathrm{~s}^{-1}$ for this object. This object shows polarization above the $3 \sigma$ limit in the $V$ and $R$ bands.

HE 0310+0059, HE 0518-2322, HE 0519-2053, HE 09150327, HE 1030-1518, HE 1345-2616, HE 1428-1950, HE 1429-1411

Medium-resolution spectral analyses and atmospheric parameters for a number of these objects can be found in the literature (Goswami 2005; Goswami et al. 2007, 2010b; Kennedy et al. 2011). The spectra of HE 0310+0059, HE 0518-2322, and HE 1056-1855 closely resemble the spectrum of HD 26, a well-known classical $\mathrm{CH}$ star (Goswami et al. 2010b). The atmospheric parameters $T_{\text {eff }}, \log g$, and metallicity $[\mathrm{Fe} / \mathrm{H}]$ for HE 0310+0059 are, respectively, $4861 \mathrm{~K}, 1.69$, and -1.32 . This object exhibits polarization above the $3 \sigma$ limit in all four bands. The atmospheric parameters for HE 0519-2053 are very similar (Table 4) to those of HE $0310+0059$; however, HE 0519-2053 shows polarization above the $3 \sigma$ limit only in the $B$ and $R$ bands. HE 0519-2053 and HE 0310+0059 both exhibit enhancement of carbon with $[\mathrm{C} / \mathrm{Fe}]$ values 0.95 and 1.24 , respectively (Kennedy et al. 2011). The object HE 1428-1950 is also enhanced in carbon with $[\mathrm{C} / \mathrm{Fe}]=1.78$. The estimated $p \%$ in $B V R I$ for HE $0310+0059$ is very similar to the estimates obtained for HE 1429-1411. The polarimetric estimates for HE 0518-2322, HE 0519-2053, HE 0915-0327, HE 0916-0037, HE 1030-1518, and HE 1345-2616 are also within a range of $0.2 \%$ to $0.9 \%$. The object HE $1428-1950$ is classified as a C-R star (Goswami et al. 2010b). The estimates of $p \%$ in $B V R I$ for this object are not very different from those of other HE stars and are within the above range.

\section{Conclusions}

$\mathrm{CH}$ and CEMP-s stars have been claimed to share similar elemental abundance properties, and this implies any possible effects of varying chemical composition on the polarization data are minimal. We used first-time $B V R I$ polarimetric observations for the sample of $\mathrm{CH}$ and CEMP stars to examine whether they also share polarimetric similarities. The polarimetric estimates of the objects are low with a range of $\sim 0.1$ to $0.9 \%$ and do not show any systematic dependence on the inverse of the effective wavelength of observation. In the plane of differential polarization, defined as the difference between the maximum and the minimum polarizations within the BVRI-bands, versus their visual magnitude, the $\mathrm{CH}$ and CEMP stars occupy a narrow band. The standard deviation for the distribution of the differential polarization is $\sim 0.036$ for the objects shown in Fig. 1 . Polarization properties of the stars can be used to infer the evolutionary stage, as well as the presence of circumstellar envelopes; expressing this in terms of their differential polarization could be useful for understanding the measure of inhomogeneity of the circumstellar dust composition. For instance, it would be interesting to examine two extreme cases of a high and a low value in differential polarization, if the high value indicated greater inhomogeneity than the lower value. It would be worthwhile examining the time dependence of this behaviour by considering a larger sample.

The objects HD 55496, HD 125079, and HD 121447 have multiple identities in the literature, as listed in the $\mathrm{CH}$ star catalogue (Bartkevicious 1996), as well as in the barium star catalogue (Lü 1991). Chemical composition studies have shown that $\mathrm{Ba}$ stars have same s-process signature as AGB stars and that they exhibit $[\mathrm{Ba} / \mathrm{Fe}]$ and $[\mathrm{Eu} / \mathrm{Fe}]$ ratios identical to those of CEMP-s stars. It was therefore suggested that the CEMP-s stars and barium stars belong to the same category of AGB masstransfer and differ only in metallicity (Allen \& Barbuy 2006b; Masseron et al. 2010). Our polarimetric estimates for these objects are not too different from the estimates of the other members of the sample.

Because both $\mathrm{CH}$ stars and the known members of CEMP-s are components of binary systems, it is reasonable to expect consequent asymmetries and inhomogeneities in the circumstellar envelopes, hence net polarization. Stars with circumstellar materials exhibit a certain amount of linear polarization. Rayleigh scattering in the stellar envelope (Harrington 1969) and dust scattering in the surrounding shells are some suggested possible mechanisms of polarization. Polarization increases with asymmetries present in the geometry of the shells. In the case of spherical symmetry, all polarization vectors cancel, and a net zero polarization is observed if the envelope is unresolved. The question of dust formation and the development of a nonspherical structure in stellar envelopes can also be addressed through polarization studies. For gaining insight into the distribution of dust, the geometry and structure of the shells, massejection mechanism, and mass-loss rates etc., it is required to conduct model-based studies of the observed polarization. Grain formation could also lead to temperature variation over the surface, causing asymmetries. In the case of cool stars, grains can condense so close to the stars that internal illunination of a dust shell by a photosphere with non-uniform surface brightness can give rise to polarization changes across a spectral band (Raveendran 1991); however, the amount of changes that can be produced by this mechanism remains to be determined.

A few objects in our sample show polarization at a level below $0.2 \%$. It would be interesting to explore how the accreted mass gets integrated into the stellar system, giving rise to a circumstellar envelope attaining perhaps spherical symmetry and thus showing null polarization. Models for mass loss with dust condensation (e.g. Winters et al. 2000; Höfner et al. 2003) assume a spherical geometry with grains forming in a shell whose radius is determined by temperature, density, and condensation 
fraction with distance from the star. Such models confirm that during dust condensation the newly formed grains absorb stellar photons and are driven outward by radiation pressure. While colliding with the molecules of the cool stellar atmospheres the grains exert an outward momentum to the gas, resulting in a dust-driven stellar wind. Observations of both the dust and gas in stellar winds show, however, that the distribution of matter is not spherical in many cases. Mapping of circumstellar envelopes in a large number of cases using maser line emission of $\mathrm{OH}$ also shows that even the most regular envelopes deviate slightly from spherical symmetry, with the wind outflow speed increasing towards the poles (Bowers 1991). Multi-epoch polarimetric observations would be useful to infer the extent of timedependent variations in polarization both in the amount and in wavelength-dependence.

Acknowledgements. We thank the anonymous referee for the useful suggestions This work made use of the SIMBAD astronomical database, operated at the CDS, Strasbourg, France, and the NASA ADS, USA. D.K. is a JRF in the DST project SR/S2/HEP-09/2007; funding from this project is gratefully acknowledged.

\section{References}

Allen, D. M., \& Barbuy, B. 2006a, A\&A, 454, 895

Allen, D. M., \& Barbuy, B. 2006b, A\&A, 454, 917

Alonso, A., Arribas, S., \& Martinez-Roger, C. 1996, A\&A, 313, 873

Bartkevicius, A. 1996, Balt. Astron., 5, 217

Beers, T. C., Chiba, M., Yoshii, Y., et al. 2000, AJ, 119, 2866

Beers, T. C., Flynn, C., Rossi, S., et al. 2007, ApJS, 168, 128

Bergeat, J., Knapik, A., \& Rutily, B. 1999, A\&A, 342, 773

Bidelman, W. P. 1981, AJ, 86, 553

Bond, H. E. 1974, ApJ, 194, 95

Bowers, P. F. 1991, ApJS, 76, 1099

Cavallo, R. M., Pilachowski, C. A., \& Rebolo, R. 1997, PASP, 109, 226

Cenarro, A. J., Peletier, R. F., Sanchez-Blazquez, P., et al. 2007, MNRAS, 374, 664

Christlieb, N., Green, P. J., Wisotzki, L., \& Reimers, D. 2001, A\&A, 375, 366

Clarke, D., Smith R. A., \& Yudin, R. V. 1998, A\&A, 336, 604

Cottrell, P. L., \& Sneden, C. 1986, A\&A, 161, 314

Dominy, J. F. 1984, ApJS, 55, 27

Dyck, H. M. 1968, AJ, 73, 688

Dyck, H. M., \& Jennings, M. C. 1971, AJ, 76, 431

Eggen, O. 1972, MNRAS, 159, 403

Francois, P. 1988, A\&A, 195, 226

Fulbright, J. P. 2000, AJ, 120, 1841

Goswami, A. 2005, MNRAS, 359, 531
Goswami, A., Bama, P., Shantikumar, N. S., \& Devassy, D. 2007, BASI, 35, 339 Goswami, A., Kartha, S. S., \& Sen, A. K. 2010a, ApJ, 722, L90

Goswami, A., Karinkuzhi, D., \& Shantikumar, N. S. 2010b, MNRAS, 402, 1111

Gratton, R. G., \& Sneden, C. 1991, A\&A, 241, 501

Gratton, R. G., \& Sneden, C. 1994, A\&A, 287, 927

Gratton, R. G., Carretta, E., \& Castelli, F. 1996, A\&A, 314, 191

Harrington, J. P. 1969, Astrophys. Lett., 3, 165

Hartwick, F. D. A., \& Cowley, A. P. 1985, AJ, 90, 2244

Höfner, S., Gautschy-Loidl, R., Aringer, B., \& Jorgensen, U. G. 2003, A\&A, 399,589

Hsu, J. C., \& Breger, M. 1982, ApJ, 262, 732

Kennedy, C. R., Thirupathi, S., Beers, T. C., et al. 2011, ApJ, 141, 102

Kruszewski, A., \& Gehrels, T. 1968, AJ, 73, 677

Lopez, J. M., \& Hiriart, D. 2011, AJ, 142, 11

Lü, P. K. 1991, AJ, 101, 2229

Lucatello, S., Tsangarides, S., Beers, T. C., et al. 2005, ApJ, 625, 833

Luck, R. E., \& Bond, H. E. 1983, ApJ, 271, L75

Luck, R. E., \& Bond, H. E. 1985, ApJ, 292, 559

Masseron, T., Johnson, J. A., Plez, B., et al. 2010, A\&A, 509, A93

McClure, R. D. 1984, ApJ, 280, L31

McClure, R. D., \& Woodsworth, A. W. 1990, ApJ, 352, 709

Melendez, J., Schuster, W. J., Silva, J. S., et al. 2010, A\&A, 522, A98

North, P. 1987, A\&A, 186, 191

North, P., \& Duquennoy, A. 1991, A\&A, 244, 335

North, P., Berthet, S., \& Lanz, T. 1994, A\&A, 281, 775N

Nissen, P. E., \& Schuster, W. J. 1997, A\&A, 326, 751

Nissen, P. E., Chen, Y. Q., Schuster, W. J., \& Zhao, G. 2000, A\&A, 353, 722

Parthasarathy, M. 1991, A\&A, 247, 429

Parthasarathy, M., Jain, S. K., \& Sarkar, G. 2005, AJ, 129, 2451

Pilachowski, C. A., Sneden, C., \& Booth, J. 1993, ApJ, 407, 699

Preston, G. W., \& Sneden, C. 2000, AJ, 120, 1014

Ramaprakash, A. N., Gupta, R., Sen, A. K., \& Tandon, S. N. 1998, A\&AS, 128, 369

Raveendran, A. V. 1991, A\&A, 243, 453

Reiz, A., \& Franco, G. A. P. 1998, A\&AS, 130, 133

Ryan, S. G., \& Lambert, D. L. 1995, AJ, 109, 2068

Sen, A. K., \& Tandon, S. N. 1994, in Instrumentation in Astronomy VIII, ed. D. L. Crawford, SPIE Proc., 2198, 1, 264

Serkowski, K. 1975, in Planets, Stars, and Nebulae studied with photopolarimetry, ed. T. Gehrels (Tuscon: Univ. Arizona Press), 135

Smith, V. V. 1984, A\&A, 132, 326

Smith, V. V., \& Lambert, D. L. 1986, ApJ, 303, 226

Smith, V. V., Coleman, H., \& Lambert, D. L. 1993, ApJ, 417, 287

Tomkin, J., Lemke, M., Lambert, D. L., \& Sneden, C. 1992, AJ, 104, 1568

Turnshek, D. A., Bohlin, R. C., Williamson, R. L., et al. 1990, AJ, 99, 1243

Vanture, A. D. 1992, AJ, 104, 1997

Winters, J. M., Le Bertre, T., Jeong, K. S., Helling, Ch., \& Sedlmayer, E. 2000, A\&A, 361, 641

Yudin, R. V., \& Evans, A. 2002, A\&A, 391, 625

Zickgraph, F.-J., \& Schulte-Ladbeck, R. E. 1989, A\&A, 214, 274 